\title{
NOTE ON CURVE AND SURFACE ENERGIES
}

\author{
JOHANNES WALLNER
}

\begin{abstract}
Energies of curves and surfaces together with their discrete variants play a prominent role as fairness functionals in geometric modeling and computer aided geometric design. This paper deals with a particular discrete surface energy which is expressible in terms of curve energies, and which occurs naturally in the problem of smoothing digital elevation data with tolerance zone constraints. We also discuss geometrically meaningful surface energies in general from the viewpoint of invariant theory, and the role of the Gauss-Bonnet theorem.
\end{abstract}

\section{A FAIRING PROBLEM WITH HARD CONSTRAINTS}

For a the polyline $p$ with vertices $p_{i}$ we consider the discrete linearized bending energy

$$
E_{2}(p)=\sum\left\|\Delta^{2} p_{i}\right\|^{2},
$$

where the forward difference operator $\Delta$ is defined by $\Delta p_{i}=p_{i+1}-p_{i}$, which implies $\Delta^{2} p_{i}=p_{i+2}-$ $2 p_{i+1}+p_{i}$. This energy functional occurs e.g. in the context of energy-minimizing curves and curve networks (cf. Hofer and Pottmann (2004) and Wallner et al. (2005)). Hofer et al. (2005) apply energy-minimizing curve networks to the problem of smoothing digital elevation data, as described below.

Suppose that a terrain is modeled by a rectangular grid of points $p_{i j}=\left(x_{i j}, y_{i j}, z_{i j}\right)$, with $i=$ $0, \ldots, N$ and $=0, \ldots, M$. The $x$ and $y$ coordinates have the form

$$
x_{i j}=i \cdot \delta_{x}, \quad y_{i j}=j \cdot \delta_{y} \quad\left(\delta_{x}, \delta_{y}>0\right) .
$$

So in fact the shape of the terrain is given only by the height field $z_{i j}$.

Terrain smoothing according to Hofer et al. (2005) starts with digital terrain data, i.e., a height field. Each vertex $p_{i j}$ is the center of its tolerance cylinder $Z_{i j}$ whose axis is parallel to the $z$-axis, and whose diameter and height typically is of the same magnitude as distances between vertices (see Fig. 1a). The problem dealt with in that paper is to compute a new height field $z_{i j}^{\prime}$ which is smoother than the original and describes a surface which passes through each tolerance cylinder. The latter condition is certainly fulfilled if $p_{i j}^{\prime}=\left(x_{i j}, y_{i j}, z_{i j}^{\prime}\right) \in Z_{i j}$, as visualized by Fig. 1b. But there is a weaker condition which still implies the required property of the new height field: It is sufficient each cylinder is met either by a 'horizontal' or a 'vertical' grid lines. In other words, it is sufficient that for all $i, j$, the cylinder $Z_{i j}$ must intersect at least one of the four line segments $p_{i j}^{\prime} p_{i-1, j}^{\prime}, p_{i j}^{\prime} p_{i+1, j}^{\prime}, p_{i j}^{\prime} p_{i, j-1}^{\prime}, p_{i j}^{\prime} p_{i, j+1}^{\prime}$. It is easy to check if this condition is met. It is illustrated in Fig. 1c. Starting from a given terrain (Fig. 2a), a fairness functional is minimized such that the constraints described above are respected. An example of the result of such a process is shown by Fig. 2. 


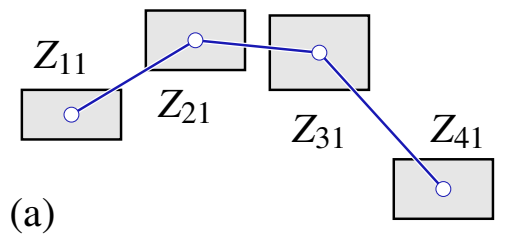

(a)

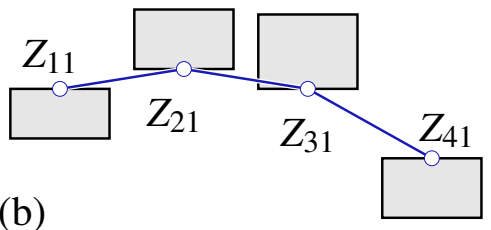

(b)

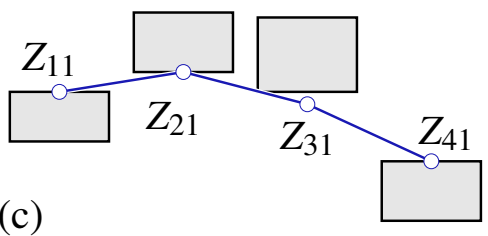

(c)

FIGURE 1. (a) Initial state before smoothing. (b) Result of smoothing with one type of constraint. (c) Result of smoothing with relaxed constraint.

(a)

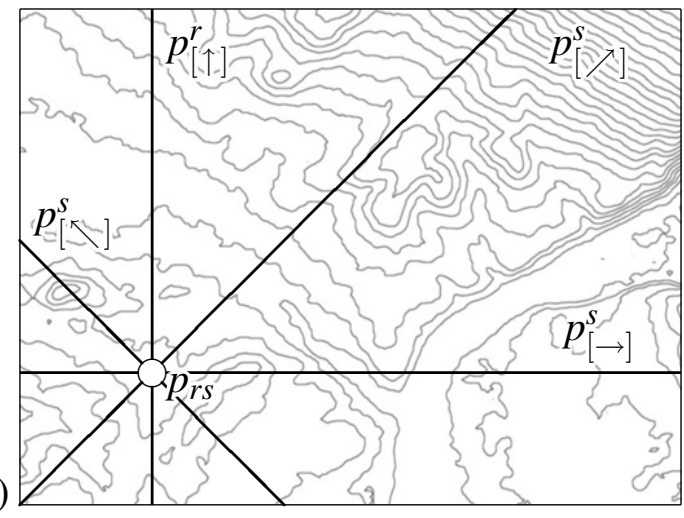

(b)

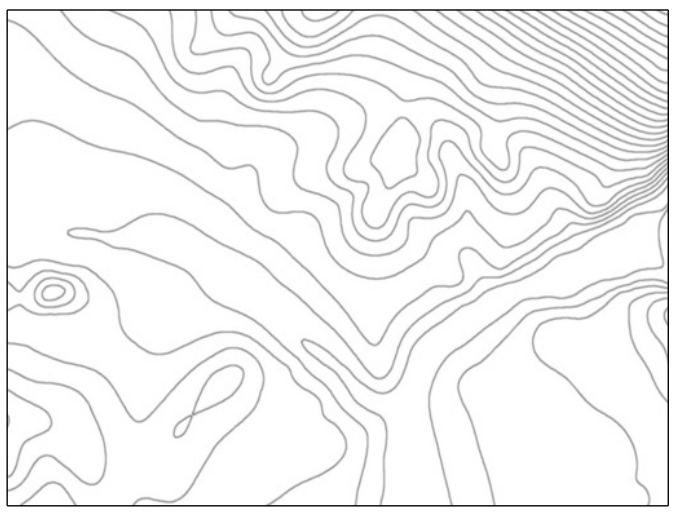

FIGURE 2. (a) Contour lines visualizing a digital terrain, represented by $400 \times 300$ grid of data points. The horizontal, vertical, and diagonal grid polylines which meet in some vertex $p_{r s}$ are shown. (b) The same model after smoothing (images taken from Hofer et al. (2005)).

The particular nature of the problem studied in Hofer et al. (2005), especially the fact that the constraints are formulated in terms of polylines, leads to fairness functionals formulated in terms of polylines lying on the discrete digital terrain model.

As above, assume that vertices $p_{i j}=\left(x_{i j}, y_{i j}, z_{i j}\right)$ with $x_{i j}$ and $y_{i j}$ as in (2) describe the terrain. We consider the horizontal grid polylines " $p_{[\rightarrow]}^{\alpha}$ ", whose vertices are defined by $\left(p_{[\rightarrow]}^{\alpha}\right)_{i}=p_{i \alpha}$. For each $\alpha$, there is such a grid polyline, which lives inside a previously defined are of interest. The total sum of energies of the horizonal grid lines is denoted by $E_{[\rightarrow]}$. In a similar way we define polylines " $p_{[\uparrow]}^{\alpha} ", " p_{[\nearrow]}^{\alpha}$ ", and " $p_{[\nwarrow]}^{\alpha}$ ":

$$
\left(p_{[\rightarrow]}^{\alpha}\right)_{i}=p_{i, \alpha}, \quad\left(p_{[\uparrow]}^{\alpha}\right)_{i}=p_{\alpha, i}, \quad\left(p_{[\nearrow]}^{\alpha}\right)_{i}=p_{\alpha+i, i}, \quad\left(p_{[\nwarrow]}^{\alpha}\right)_{i}=p_{\alpha-i, i}
$$

The total sums of energies of the vertical and the two types of diagonal grid polylines are denoted by they symbols $E_{[\uparrow]}, E_{[\ltimes]}$, and $E_{[\nearrow]}$, respectively. It is one of the purposes of this paper to show why

$$
E=E_{[\rightarrow]}+E_{[\uparrow]}+\frac{1}{4}\left(E_{[\nwarrow]} E_{[\nearrow]}\right)
$$

is a geometrically meaningful discrete surface energy. 


\section{Differential Geometry Facts}

Here we summarize some facts of Differential Geometry, whose proofs can be found in do Carmo (1976). Suppose a surface in Euclidean space contains a domain $B$ with piecwise $C^{2}$ boundary curve $c$ of length $L$. An arc length parametrization of $c$ is indicated by writing " $c(s)$ ". Each point $p$ of the surface has a unit normal vector $n(p)$. The mapping $p \mapsto n(p)$ is smooth, if the surface is $C^{2}$. Rotation by 90 degrees in the tangent plane of $p$ is effected by $X \mapsto X^{\perp}=n(p) \times X$. The two principal curvatures, Gaussian curvature, and mean curvatures in a point $p$ of the surface under consideration are denoted by $\kappa_{1}(p), \kappa_{2}(p), K(p)$ and $H(p)$, respectively.

2.1. Angular velocities. Recall that the angular velocity of a time-dependent unit vector $X(t)$ in the Euclidean plane is given by $\dot{\varangle} X(t)=\left\langle\dot{X}, X^{\perp}\right\rangle$, where $\langle u, v\rangle$ denotes the scalar product of the vectors $u, v$, and the dot indicates differentiation. If $c(t)$ is a curve in a given surface and $X(t)$ is a unit vector tangent to the that surface in the point $c(t)$, then this vector field's angular velocity and total turning angle in the interval $t \in[a, b]$ are defined by

$$
\dot{\varangle} X:=\left\langle\dot{X}, X^{\perp}\right\rangle=\operatorname{det}(X, \dot{X}, n \circ c), \quad \varangle_{a}^{b} X(t)=\int_{a}^{b} \dot{\varangle} X(t) d t .
$$

The geometric interpretation of $\dot{\varangle}$ is as follows: If $Y(t)$ is a unit vector field along the same curve obtained by geodesic parallel transport, such that $X=\cos \phi \cdot Y+\sin \phi \cdot Y^{\perp}$, then $\dot{\phi}=\dot{\varangle} X$. The usage of the symbol " $\varangle X(t)$ " implies knowledge of of the surface's normal vector in the point which $X(t)$ it is attached to. It is elementary that the total turning angle is invariant in the following sense: If $t^{\prime}=f(t)$ is a transformation of the curve parameter and $X^{\prime}\left(t^{\prime}\right):=X(f(t))$ is the transformed vector field, then $\varangle_{a}^{b} X=\varangle_{a^{\prime}}^{b^{\prime}} X^{\prime}$.

2.2. Geodesic curvature. The geodesic curvature $\kappa_{g}(s)$ of the curve $c(s)$ is the angular velocity of $\dot{c}(s)$ when $c$ is parametrized by arc length: $\kappa_{g}(s)=\dot{\varangle} \dot{c}(s)=\operatorname{det}(\dot{c}(s), \ddot{c}(s), n(c(s)))$. There are only finitely many parameter values $s=s_{1}, s_{2}, \ldots$ where the curve $c$ is not twice differentiable, and where the tangent vector $\dot{c}$ possibly jumps. The angle of this jump, measured with respect to the orientation provided by the surface's normal vector $n(c(s))$, is denoted by $\varangle_{s}^{s} \dot{c}$. We assume that arc length parametrization of $c$ is such that the vector $n(c(s)) \times \dot{c}(s)$ points towards the inside of $B$. Suppose a unit vector field $Y$ is defined globally in $B$ and even a bit outside $B$ (i.e., in a neighbourhood of $B \cup \partial B)$. Then the following equation holds:

$$
\varangle_{0}^{L} Y(s)=\oint_{c} \dot{\varangle} Y(s)=\oint_{c} \kappa_{g}(s) d s+\sum_{s \in[0, L)} \varangle_{s}^{s}(\dot{c})-2 \pi .
$$

Here the $\oint_{c}$ sign means that we integrate along the entire closed curve $c$, e.g. from $s=0$ to $s=L$. It is important that both the geodesic curvature and the turning angles use only first derivatives of the surface, viz., the normal vectors.

2.3. Willmore energy and the Gauss-Bonnet formula. For the domain $B$, its boundary curve $c=\partial B$, and any globally defined vector field $Y$ as above, we have the famous integral formula of Gauss-Bonnet, which relates the surface integral of Gaussian curvature with a curve integral.

$$
\int_{B} K=-\varangle_{0}^{L}(Y)
$$


The form which is usually given is retrieved from (7) via (6). The symbol $\int_{B}$ means the surface area integral. As is well known, (7) can be used for identifying a "boundary component" in quadratic energy functionals involving the principal curvatures. An example of this is the Willmore energy (see e.g. Clarenz et al. (2004)):

$$
W(B)=\int_{B}\left(\frac{1}{2}\left(\kappa_{1}-\kappa_{2}\right)\right)^{2}=\int_{B}\left(H^{2}-K\right)=\int_{B} H^{2}+\varangle_{0}^{L}(Y) .
$$

Here $Y$ is an arbitrary globally defined vector field, as in (7). If the curve $c$ is considered fixed, while the surface is subject to variation, this splitting identifies a part of the Willmore energy which depends on the actual second derivates of the surface (the $\int H^{2}$ part), and a part which solely depends on the surface's normal vectors along $\partial B$ (the $\int K$ part).

\section{GRAPH SURFACES}

We now consider a surface which is the graph of a $C^{3}$ function $f$ defined in an open subset of $\mathbb{R}^{2}$. This means that the surface is parametrized by $g(u, v)=(u, v, f(u, v))$. It is easy to compute normal vector $n$, Gaussian curvature $K$, and mean curvature $H$ for such graph surfaces. The result is given below, after some notation has been introduced. Differentiation with respect to $u$ and $v$ is indicated by subscripts.

$$
\begin{aligned}
& D:=1+f_{, u}^{2}+f_{, v}^{2}, \quad n(u, v)=D^{-1 / 2}\left[-f_{, u},-f_{, v}, 1\right], \quad \operatorname{Hess}(f):=\left[\begin{array}{ll}
f_{, u u} & f_{, u v} \\
f_{, u v} & f_{, v v}
\end{array}\right], \\
& K=D^{-2} \operatorname{det} \operatorname{Hess}(f), \quad H=\frac{1}{2} D^{-3 / 2}\left(\Delta f+f_{, u u} f_{, v}^{2}-2 f_{, u} f_{, v} f_{, u v}+f_{, v v} f_{, u}^{2}\right) .
\end{aligned}
$$

Later we use the vector field $Y=g_{, u} /\left\|g_{, u}\right\|$, which expands to $Y=\left(1+f_{, u}^{2}\right)^{-\frac{1}{2}}\left[1,0, f_{, u}\right]$.

3.1. First order approximation for almost flat surfaces. In view of the applications considered below, we are mainly interested in the case the first derivatives of $f$ are small:

$$
f_{, u} \ll 1, \quad f_{, v} \ll 1 \quad \Longrightarrow \quad f_{, u}^{2} \approx 0, \quad f_{, u} f_{, v} \approx 0, \quad f_{, v}^{2} \approx 0 ;
$$

but we don't require the same of the second derivatives. The reason why we consider that particular type of surface is that we are going to use graph surfaces as mathematical description of a piece of topography, which for large parts of the earth is rather flat.

The exact meaning of the symbol " $\approx$ " is that of equality up to an additive constant of magnitude $o\left(\sqrt{f_{, u}^{2}+f_{, v}^{2}}\right)$, or more precisely, $O\left(f_{, u}^{2}+f_{, v}^{2}\right)$ as $f_{, u}, f_{, v} \rightarrow 0$. In order to compute the angular velocity of $Y$ along the curve $c$, we evaluate $\dot{Y}=\dot{u} Y_{, u}+\dot{v} Y_{, v}$ and compute $\operatorname{det}(Y, \dot{Y}, n)$ :

$$
Y_{, u} \approx\left[-f_{, u} f_{, u u}, 0, f_{, u u}\right], Y_{, v} \approx\left[-f_{, u} f_{, u v}, 0, f_{, u v}\right], \dot{\varangle}(Y) \approx \dot{u} f_{, v} f_{, u u}+\dot{v} f_{, v} f_{, u v} .
$$

According to (10), Gaussian and mean curvature are approximated by

$$
K \approx \bar{K}=f_{, u u} f_{, v v}-f_{, u v}^{2}=\operatorname{det} \operatorname{Hess}(f), \quad H \approx \bar{H}=\frac{1}{2}\left(f_{, u u}+f_{, v v}\right)=\frac{1}{2} \Delta f .
$$

We use the surface parametrization " $g$ " to define the domain $\bar{B}$ and its boundary $\bar{c}$ in $\mathbb{R}^{2}$ by $B=g(\bar{B})$ and $c(s)=g(\bar{c}(s))$, and rewrite the surface integral of the Gaussian curvature in a form which allows 
to apply Green's theorem:

$$
\int_{B} K=\int_{\bar{B}} \frac{\operatorname{det} \operatorname{Hess}(f)}{\Delta^{3 / 2}} d u d v \approx \int_{\bar{B}} \bar{K} d u d v=\int_{\bar{B}}\left(\left(f_{, u u} f_{, v}\right)_{, v}-\left(f_{, u v} f_{, v}\right)_{, u}\right) d u d v .
$$

This expression can now be converted into a curve integral:

$$
\int_{\bar{B}} \bar{K} d u d v=-\oint_{\bar{c}} f_{, u u} f_{, v} d u+f_{, u v} f_{, v} d v=-\oint_{\bar{c}} f_{, v} d\left(f_{, u} \circ c\right) .
$$

This is exactly the first order approximant of the Gauss-Bonnet formula (7), if we adopt the first order approximants of $K$ and $\dot{\varangle}(Y)$ from above. $Y$ was rather arbitrary, except that for different $Y$ 's Green's theorem may yield different approximations for the turning angle. In case the support of $f$ is contained in $\bar{B}$, we have $f_{, u}=f_{, v}=0$ for all boundary points. This results in $\int_{B} K=0, W(B)=\int_{B} H^{2}$, $\int_{\bar{B}} \bar{K} d u d v=0$.

3.2. Curve energies. The curve $c: I \rightarrow \mathbb{R}^{3}$ has the linearized bending energy

$$
E_{2}(c)=\int_{I}\|\ddot{c}(t)\|^{2} d t
$$

It is well known that minimizers of $E_{2}$ under interpolation conditions are the cubic B-spline curves. We are interested in the energies of special curves contained in a graph surface with parametrization $g(u, v)=(u, v, f(u, v))$ : Assume that $c(t)=g\left(u_{0}+a t, v_{0}+b t\right)$. It follows that the energy of such a curve can be written as

$$
E_{2}(c)=\left.\int_{I}[a, b] \cdot \operatorname{Hess}(f)\right|_{\substack{u=u_{0}+a t \\
v=v_{0}+b t}} \cdot\left[\begin{array}{l}
a \\
b
\end{array}\right] d t .
$$

Later we establish a connection between curve energies and surface energies, where curves of that special form occur.

3.3. Invariants of the Hessian. A different choice of a Cartesian coordinate system in the parameter domain causes a change of coordinates $(u, v) \mapsto\left(u^{\prime}, v^{\prime}\right)$ and consequently $f$ transforms to $f^{\prime}\left(u^{\prime}, v^{\prime}\right):=f\left(u\left(u^{\prime}, v^{\prime}\right), v\left(u^{\prime}, v^{\prime}\right)\right)$ :

$$
\left[\begin{array}{l}
u^{\prime} \\
v^{\prime}
\end{array}\right]=M\left[\begin{array}{l}
u \\
v
\end{array}\right]+\left[\begin{array}{l}
u_{0} \\
v_{0}
\end{array}\right], M=\left[\begin{array}{rr}
\cos \phi & -\sin \phi \\
\sin \phi & \cos \phi
\end{array}\right] .
$$

The Hessian changes as follows:

$$
\operatorname{Hess}\left(f^{\prime}\right)\left(u^{\prime}, v^{\prime}\right)=M^{T} \operatorname{Hess}(f)(u, v) M .
$$

It is well known that trace and determinant (or equivalently, the unordered pair of eigenvalues) constitute a complete system of invariants with respect to the planar rotation group when acting in this way on symmetric $2 \times 2$ matrices. Moreover it is easy to show that $\left\{\operatorname{tr}^{2}\right.$, det $\}$ is a basis of the linear space of quadratic homogeneous invariant polynomials in the three indeterminates $f_{, u u}, f_{, u v}, f_{, v v}$. In view of (Equ. 13), also the approximate curvatures can be used to give a basis $\left\{\bar{H}^{2}, \bar{K}\right\}$ of that space. This is summarized by the following theorem, whose proof, for the convenience of the reader, is repeated in section 5 . 
Theorem 1. A polynomial $p$ defined in the space of symmetric $n$ by $n$ matrices with the property that $p\left(g^{T} x g\right)=p(x)$ for all orthogonal matrices $g$ is expressible as a polynomial in the coefficients of the characteristic polynomial of $x$.

It follows that for 2 by 2 matrices, any such invariant polynomial is expressible in terms of determinant and trace.

3.4. Combined curve energies. The following theorem expresses surface energies in terms of curve energies. Because the surface energy itself is an area integral, we cannot expect that a finite number of curves defines a surface energy. Rather, we consider families of curves which cover the surface under consideration, and integrate over the energies computed from each of them. Th. 2 is the basis of a discretization discussed in Section 4.

Theorem 2. Assume that $\bar{B}$ is a planar domain and $f$ is a $C^{2}$ function as above. Consider the surface parametrization $g(u, v)=(u, v, f(u, v))$, and its approximate curvatures $\bar{K}(u, v)$ and $\bar{H}(u, v)$ according to (13). For each $\alpha \in \mathbb{R}$, there are curves $\bar{c}_{1, \alpha}(t)=g(t, \alpha), \bar{c}_{2, \alpha}(t)=g(\alpha, t), \bar{c}_{3, \alpha}(t)=$ $g(\alpha+t, t)$, and $\bar{c}_{4, \alpha}(t)=g(\alpha+t,-t)$. These curves comprise the mesh of the $u$ and $v$ parameter lines, together with the diagonals of that mesh. Then

$$
8 \int_{\bar{B}}\left(3 \bar{H}^{2}-\bar{K}\right) d u d v=\int_{\alpha}\left(4 E_{2}\left(c_{1, \alpha}\right)+4 E_{2}\left(c_{2, \alpha}\right)+E_{2}\left(c_{3, \alpha}\right)+E_{2}\left(c_{4, \alpha}\right)\right) d \alpha .
$$

Each curve energy is computed by integration over that part of the real axis which corresponds to the points contained in B. Up to a common factor, this is the only linear combination of the energies of curves $c_{i, \alpha}$ which results in an invariant surface energy.

Proof. We expand the right hand side of (20) according to (17) and make the following changes of variables in the four respective integrals: $(t, \alpha)=(u, v),(\alpha, t)=(u, v),(\alpha+t, t)=(u, v),(\alpha+$ $t,-t)=(u, v)$. We get

$$
\begin{aligned}
\text { r.h.s. } & =4 \int_{\alpha}\left(\int_{t} f_{, u u}(t, \alpha)^{2} d t\right) d \alpha+4 \int_{\alpha}\left(\int_{t} f_{, v v}(\alpha, t)^{2} d t\right) d \alpha \\
& +\int_{\alpha}\left(\left.\int_{t}\left(f_{, u u}+2 f_{, u v}+f_{, v v}\right)\right|_{\left(\begin{array}{c}
(u, v)= \\
(\alpha+t, t)
\end{array}\right.} ^{2} d t\right) d \alpha+\int_{\alpha}\left(\left.\int_{t}\left(f_{, u u}-2 f_{, u v}+f_{, v v}\right)\right|_{\left(\begin{array}{c}
(u, v)= \\
(\alpha+t, t)
\end{array}\right.} ^{2} d t\right) d \alpha \\
& =\int_{\bar{B}}\left(4 f_{, u u}^{2}+4 f_{, v v}^{2}+\left(f_{, u u}+2 f_{, u v}+f_{, v v}\right)^{2}+\left(f_{, u u}-2 f_{, u v}+f_{, v v}\right)^{2}\right) d u d v \\
& =\int_{\bar{B}}\left(24 \bar{H}^{2}-8 \bar{K}\right) d u d v .
\end{aligned}
$$

The last equality follows from the definition of $\bar{H}$ and $\bar{K}$. Now we consider the uniqueness question. We ask which linear combations of the form $\sum \lambda_{i} E_{2}\left(c_{i, \alpha}\right)$ yield a meaningful surface energy, i.e., one whose definition is invariant under rotation of the coordinate system. In view of Th. 1 , this boils down to the question which linear combinations of the form $\sum \lambda_{i}\left\|c_{i}\right\|^{2}$ are also linear combinations of $\bar{K}$ and $\bar{H}^{2}$. We express the norms of second derivatives of curves in terms of the Hessian:

$$
\left\|\ddot{c}_{1}\right\|^{2}=f_{, u u}^{2}, \quad\left\|\ddot{c}_{2}\right\|^{2}=f_{, v v}^{2}, \quad\left\|\ddot{c}_{3,4}\right\|^{2}=\left(f_{, u u} \pm 2 f_{, u v}+f_{, v v}\right)^{2} .
$$


Then we solve the equation

$$
\begin{aligned}
& \sum_{i=1}^{4} \lambda_{i}\left\|\ddot{c}_{i}\right\|^{2}=\sigma \bar{H}^{2}+\tau \bar{K} \quad \Longleftrightarrow \lambda_{1} f_{, u u}^{2}+\lambda_{2} f_{, v v}^{2}+\lambda_{3}\left(f_{, u u}+2 f_{, u v}+f_{, v v}\right)^{2} \\
& +\lambda_{3}\left(f_{, u u}-2 f_{, u v}+f_{, v v}\right)^{2}=\sigma\left(f_{, u u}+f_{, v v}\right)^{2} / 4+\tau\left(f_{, u u} f_{, v v}-f_{, u v}^{2}\right) .
\end{aligned}
$$

Its solution, as stated in the theorem, is given by $\left[\lambda_{1}, \ldots, \lambda_{4}, \sigma, \tau\right]=t[4,4,1,1,-24,8](t \in \mathbb{R})$.

\section{Discretization}

Th. 2 shows that a certain integral of curve energies yields a geometrically meaningful surface energy. It is easy to discretize that integral: A discretization of the linearized bending energy of (16) is given by (1). Integrating over $d \alpha$ in (20) is discretized by taking polylines which correspond to equidistant values of $\alpha$. For a regular height field as described in the beginning, this results in summing up the curve energies of horizontal, vertical, and diagonal grid lines as already written down in (4). In explicit form, the discrete fairness functional reads

$$
\begin{aligned}
E=\sum_{i, j} & \left(\left(z_{i, j+1}-2 z_{i j}+z_{i, j-1}\right)^{2}+\left(z_{i+1, j}-2 z_{i j}+z_{i-1, j}\right)^{2}\right. \\
& \left.+\frac{1}{4}\left(z_{i+1, j+1}-2 z_{i j}+z_{i-1, j-1}\right)^{2}+\frac{1}{4}\left(z_{i+1, j-1}-2 z_{i j}+z_{i-1, j+1}\right)^{2}\right),
\end{aligned}
$$

Here an index pair $i, j$ occurs only if all index pairs which contribute to the sum are within range.

\section{APPENDIX: QUADRATIC INVARIANTS OF SYMMETRIC MATRICES}

In this paper we use properties of the Hessian of a $C^{2}$ function which are invariant with respect to a change of the Cartesian coordinate system in use. We are especially interested in polynomials of the Hesse matrix which have this property. The well known result of Th. 1 gives the required answers. Its proof on the basis of properties of elementary symmetric functions is shown below.

Proof. (of Th. 1) We generally use the notation $\mathbb{R}[V]$ for the ring of real polynomials defined in the vector space $V$, and $\mathbb{R}\left[X_{1}, \ldots, X_{n}\right]$ if $X_{i}$ denote coordinates in such a space. The latter notation is used if we want to explicitly write $p\left(X_{1}, \ldots, X_{n}\right)$ for a polynomial contained in that polynomial ring. The characteristic polynomial of a symmetric matrix $x$ reads $\operatorname{det}\left(x-t E_{n}\right)=\sum_{j=0}^{n} a_{j}(x) t^{j}$, which defines the coefficient polynomials $a_{j} \in \mathbb{R}\left[M_{\text {symm }}\right]$. For instance, we have $a_{0}(x)=\operatorname{det} x$. For a diagonal matrix $x=\operatorname{diag}\left(t_{1}, \ldots, t_{n}\right)$, it is elementary that $a_{j}(x)=(-1)^{j} s_{n-j}\left(t_{1}, \ldots, t_{n}\right)$, where $s_{i} \in \mathbb{R}\left[t_{1}, \ldots, t_{n}\right]$ is the $i$-th elementary symmetric function of $n$ variables:

$$
\begin{aligned}
& s_{0}\left(t_{1}, \ldots, t_{n}\right)=1, \quad s_{1}\left(t_{1}, \ldots, t_{n}\right)=t_{1}+\cdots+t_{n}, \\
& s_{2}\left(t_{1}, \ldots, t_{n}\right)=\sum_{i<j} t_{i} t_{j}, \ldots s_{n}\left(t_{1}, \ldots, t_{n}\right)=t_{1} \cdots t_{n} .
\end{aligned}
$$

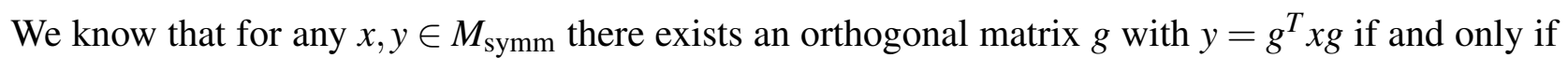
the characteristic polynomials of $x$ and $y$ agree. It follows that $a_{0}, \ldots, a_{n}$ are invariant polynomials.

Now assume that $p \in \mathbb{R}\left[M_{\text {symm }}\right]$ is invariant. We define $q \in \mathbb{R}\left[t_{1} \ldots, t_{n}\right]$ by $q\left(t_{1}, \ldots, t_{n}\right)=p\left(\operatorname{diag}\left(t_{1}\right.\right.$, $\left.\left.\ldots, t_{n}\right)\right)$. For any permutation $\sigma=(\sigma(1), \ldots, \sigma(n))$ of the numbers $(1, \ldots, n)$, there is an orthogonal matrix $s$ such that for all vectors $\left(t_{1}, \ldots, t_{n}\right)^{T}$, we have $\left(t_{\sigma(1)}, \ldots, t_{\sigma(n)}\right)^{T}=s \cdot\left(t_{1}, \ldots, t_{n}\right)^{T}$. Then, 
$s^{T} \operatorname{diag}\left(t_{1}, \ldots, t_{n}\right) s=\operatorname{diag}\left(t_{\sigma(1)}, \ldots, t_{\sigma(n)}\right)$. We see that permuting the diagonal elements by $\sigma$ is effected by the action of an orthogonal matrix. As $p$ was assumed invariant, $q$ is invariant with respect to permutations of the variables $t_{1}, \ldots, t_{n}$. Sturmfels (1993) gives an algorithm to rewrite $q$ as a polynomial in the elementary symmetry functions, i.e., there is $r \in \mathbb{R}\left[\mathbb{R}^{n+1}\right]$ such that

$$
q\left(t_{1}, \ldots, t_{n}\right)=r\left(s_{0}\left(t_{1}, \ldots, t_{n}\right), \ldots, s_{n}\left(t_{1}, \ldots, t_{n}\right)\right) .
$$

We define the polynomial $p^{\prime} \in \mathbb{R}\left[M_{\text {symm }}\right]$ by

$$
p^{\prime}(x)=r\left(a_{n}(x),-a_{n-1}(x), \ldots,(-1)^{n} a_{0}(x)\right),
$$

which is invariant by construction, and it is chosen such that in case $x$ is a diagonal matrix, $p(x)=$ $p^{\prime}(x)$. For every $x \in M_{\text {symm }}$ there is an orthogonal matrix $g$ such that $g^{T} x g$ is a diagonal matrix. It follows that $p(x)=p\left(g^{T} x g\right)=p^{\prime}\left(g^{T} x g\right)=p^{\prime}(x)$. These equalities follow, in turn, from invariance of $p$, the previous observation, and invariance of $p^{\prime}$. This concludes the proof of Th. 1.

\section{ACKNOWLEDGMENTS}

This research was supported by the Austrian Science Foundation (FWF) under grant number P15911.

\section{REFERENCES}

Clarenz, U., Diewald, U., Dziuk, G., Rumpf, M., Rusu, R., 2004. A finite element method for surface restoration with smooth boundary conditions. Comput. Aided Geom. Design 21, 427-445.

do Carmo, M. P., 1976. Differential Geometry of Curves and Surfaces. Prentice-Hall, New York.

Hofer, M., Pottmann, H., 2004. Energy-minimizing splines in manifolds. ACM Trans. Graphics 23 (3), 284-293, Proc. ACM SIGGRAPH 2004.

Hofer, M., Sapiro, G., Wallner, J., August 2005. Fair polyline networks for constrained smoothing of digital terrain elevation data. IMA Preprint 2058, Univ. Minnesota.

URL http: //www.geometrie.tuwien.ac.at/wallner/dted.pdf

Sturmfels, B., 1993. Algorithms in invariant theory. Texts and Monographs in Symbolic Computation. Springer-Verlag, Vienna.

Wallner, J., Pottmann, H., Hofer, M., January 2005. Fair webs. Tech. Rep. 134, Geometry Preprint Series, Vienna Univ. of Technology.

URL http: //www .geometrie.tuwien.ac .at/wallner/fairwebs . pdf

Vienna University of Technology, institute of Discrete Mathematics and Geometry. Email WALLNER@GEOMETRIE.TUWIEN.AC.AT. 